\title{
Fecal immunochemical testing results and characteristics of colonic lesions
}

Authors

Institutions
Sascha C. van Doorn ${ }^{1}$, Inge Stegeman ${ }^{2}$, An K. Stroobants ${ }^{3}$, Marco W. Mundt ${ }^{4}$, Thomas R. de Wijkerslooth' Paul Fockens $^{1}$, Ernst J. Kuipers ${ }^{5}$, Patrick M. Bossuyt ${ }^{2}$, Evelien Dekker ${ }^{1}$

Institutions are listed at the end of article. submitted 20. August 2014 accepted after revision 1. April 2015

\section{Bibliography}

Dol http://dx.doi.org/ 10.1055/s-0034-1392412

Published online: 30.6 .2015 Endoscopy 2015; 47: 10111017

(c) Georg Thieme Verlag KG Stuttgart · New York ISSN 0013-726X

\section{Corresponding author}

Evelien Dekker, MD, PhD Department of

Gastroenterology and Hepatology

Academic Medical Center

Meibergdreef 9

1105 AZ, Amsterdam

The Netherlands

Fax: +31-20-6917033

e.dekker@amc.uva.nl
Background and study aims: Fecal immunochemical tests (FIT) are used to detect blood in feces, which might indicate the presence of colorectal neoplasia. The aim of this study was to investigate whether FIT results vary depending on the characteristics of colonic lesions.

Patients and methods: This was a retrospective analysis of lesions detected in a cohort of asymptomatic individuals (aged 50-75 years) who were invited to participate in a FIT-based screening pilot in The Netherlands. The mean FIT result was compared across subgroups of individuals defined by histopathology of the most advanced lesion detected. In addition, the results were compared with data from a primary colonoscopy screening trial, in which participants also completed a FIT.

Results: In three rounds of FIT-based screening, a total of 877 FIT-positive individuals underwent colonoscopy. Higher mean FIT results (hemoglo-

\section{Introduction \\ $\nabla$}

Colorectal cancer (CRC) is the second most common type of cancer in Western countries, and almost half of those diagnosed die from the disease [1]. The clinical and pathological stage at the time of diagnosis largely determines prognosis. Population screening for CRC aims to detect cancer and precancerous lesions at an early stage, and to reduce morbidity and mortality of the disease [2]. The fecal immunochemical test (FIT) is a quantitative fecal occult blood test that is specific for human blood and is used as a screening tool [3-7]. CRCs with a higher $\mathrm{T}$ stage are associated with higher FIT results [8]. Several trials have also reported significantly higher fecal hemoglobin levels in patients with advanced neoplasia than in patients with a normal colonoscopy $[4,5,9,10]$. Further evaluation of the association between lesion characteristics and absolute FIT results (measured in $\mu \mathrm{g} \mathrm{Hb} / \mathrm{g}$ feces) in a screening popu- bin $[\mathrm{Hb}] / \mathrm{g}$ feces) were observed in individuals

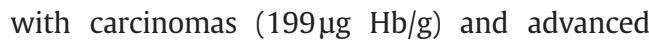
adenomas $(87 \mu \mathrm{g} \mathrm{Hb} / \mathrm{g})$ compared with participants with nonadvanced adenomas $(50 \mu \mathrm{g} \mathrm{Hb} / \mathrm{g})$ or those with serrated lesions $(46 \mu \mathrm{g} \mathrm{Hb} / \mathrm{g}$ ) $(P<0.001)$. In the primary colonoscopy trial, 1256 participants completed a FIT test and underwent colonoscopy. The number of participants with nonadvanced adenomas as the most advanced lesion was comparable between this group and the FIT-based screening group (20\% vs. $22 \%$ ).

Conclusion: In FIT-based screening, the mean FIT results varied depending on the characteristics of the most advanced colonic lesion. The proportion of participants with a nonadvanced adenoma as the most advanced lesion was similar in the FITbased screening group and in the primary colonoscopy screening group, suggesting that these lesions are coincidental findings rather than FITdetected findings.

lation would improve the understanding of the performance of FIT. It would be useful to know which lesions are detected during FIT-based screening and which lesions are coincidental findings on colonoscopy, as this would help to develop other, more sensitive, screening tests or screening strategies. One recent study reported more positive FIT test results in individuals with more advanced lesions [11], and Digby et al. demonstrated an association between fecal hemoglobin and the severity of neoplastic disease in a large FIT-positive cohort undergoing colonoscopy [12].

The current study aimed to confirm these findings by comparing fecal hemoglobin concentrations obtained in FIT-based screening across subgroups defined by the most advanced lesion detected on subsequent colonoscopy. In addition, the findings in FIT-positive individuals were compared with results from a primary colonoscopy screening trial, performed in The Netherlands, in 
which participants also completed a FIT. In the latter study group, all participants underwent colonoscopy, regardless of their FIT result. This allowed the number and histopathology of lesions in FIT-positive individuals from FIT-based screening to be compared with findings from an asymptomatic primary colonoscopy screening group. The incidence of nonadvanced lesions was of interest, as the FIT results of these lesions are unknown.

\section{Patients and methods}

$\nabla$

\section{Population and design}

Data from pilot programs of FIT-based screening and from primary colonoscopy screening, collected from comparable regions in The Netherlands, were used in the study.

\section{FIT-based screening}

Data from FIT-based screening were collected in three rounds of a biennial FIT-based screening pilot in the Amsterdam region of The Netherlands. The study designs of the three consecutive screening rounds have been reported in detail elsewhere $[4,13$ 15]. In brief, ethical approval was obtained from the Dutch Health Council (2005/03WBO, The Hague, The Netherlands). In each of three screening rounds, asymptomatic, average-risk, individuals aged between 50 and 75 years were invited to participate (see - Fig. e1, available online). This FIT-based pilot screening program was designed as a dynamic cohort study; individuals included in the first round were re-invited in subsequent rounds, as were individuals who had moved into the target area, and those who had reached the target age between screening rounds. Individuals who had moved out of the area or exceeded the age limit, or who had had a previous positive FIT result were not reinvited. Institutionalized people were excluded from participation. Eligible persons were identified using the electronic database of the regional municipal administration registration. Preannouncements and invitations were sent out by the regional Foundation of Population Screening Mid-West using a centralized invitation program (ICOLON).

Only those who underwent a colonoscopy after a positive FIT test result were included in the present analyses. FIT-positive screening individuals were not invited for further FIT-based screening rounds and were therefore included only once in the analyses.

\section{Colonoscopy-based screening}

In the primary colonoscopy screening trial, asymptomatic, average-risk participants, who were in the same age group as individuals in the FIT-based pilot screening program, were randomly allocated to an invitation for primary CRC screening by colonoscopy or screening by computed tomography colonography (see - Fig. e2, available online). Ethical approval was obtained from the Dutch Health Council (2009/03WBO, The Hague, Netherlands). All participants gave written informed consent. The design and results of this randomized controlled trial (COCOS-Colonoscopy Or CT colonography Screening) have been described in detail elsewhere $[14,16-18]$. Only participants from the colonoscopy arm were eligible for the analyses in the current study. Between June 2009 and July 2010, a total of 6600 individuals from the Amsterdam and Rotterdam regions of The Netherlands were randomly selected from the municipal administration registrations and invited for colonoscopy screening. Screening participants who were willing to undergo colonoscopy were additionally invited to complete one sample FIT before their screening colonoscopy. The FIT results of those who were allocated to and underwent primary colonoscopy and who provided written informed consent were included in the current analyses.

\section{Stool tests}

The same FIT test (OC Sensor; Eiken Chemical Co., Tokyo, Japan) was used for all three FIT-based screening rounds and for the primary colonoscopy screening trial. This test provides a quantitative measurement of fecal human hemoglobin. The FIT consists of a probe attached to a cap, which fits a collection tube containing hemoglobin-stabilizing buffer. In the Laboratory for General Clinical Chemistry for special techniques at the Academic Medical Center, Amsterdam, which is certified according to CCKL (ISO 9001), the returned samples from both studies were analyzed using the OC Sensor automated instrument.

The cut-off value for FIT positivity in the current study was $10 \mu \mathrm{g}$ $\mathrm{Hb} / \mathrm{g}$ feces, which corresponds to $50 \mathrm{ng} \mathrm{Hb} / \mathrm{mL}$ buffer [7].

\section{FIT-based screening}

In FIT-based screening, an invitation kit containing the FIT was sent by postal mail. In addition to the invitation letter, the kit also contained a detailed information brochure, a test instruction leaflet, and a return envelope. A signed informed consent form had to be enclosed in the return envelope. Participants could perform the test at home and return it by postal mail.

\section{Colonoscopy-based screening}

In the primary colonoscopy screening trial, screening participants received extensive, verbal instructions on performing the FIT during a consultation prior to the colonoscopy. They were asked to perform the FIT at home, within 48 hours prior to colonoscopy, but before starting the bowel preparation. Participants were asked to bring the FIT to the screening center, or to call the screening center immediately after performing the FIT, so that research staff could come and collect the FIT sample.

\section{Colonoscopy}

In FIT-based screening, colonoscopy was recommended only for individuals with a positive FIT test result. In the primary colonoscopy screening trial, all screenees underwent colonoscopy, regardless of their FIT result. The endoscopists who performed the colonoscopies were blinded to the FIT result.

Participants eligible for colonoscopy were invited for a consultation at one of the screening centers. Individuals from the FITbased screening rounds who had undergone a colonoscopy in the 2 years prior to the positive FIT result were advised not to undergo a repeat colonoscopy. In the first two rounds, all colonoscopies were performed at the Academic Medical Center in Amsterdam by approximately 30 endoscopists. In the third round, colonoscopies were also performed at the Flevoziekenhuis in Almere by two endoscopists. Colonoscopies within the COCOS trial were performed at the Academic Medical Center in Amsterdam and in the Erasmus Medical Center in Rotterdam by five endoscopists. Colonoscopy quality indicators were recorded on a case record form. When polyps were detected, endoscopic removal of the lesions was attempted during the same procedure. If cancer was suspected or if endoscopic removal was not possible, biopsies were obtained and histopathological assessment provided a definitive diagnosis to inform further treatment decisions. 


\section{Histopathology}

All lesions were evaluated by an experienced gastrointestinal pathologist according to the Vienna criteria [19]. Lesions were classified as follows: adenocarcinoma, adenoma (tubular, tubulovillous, villous), hyperplastic, sessile serrated adenoma/polyp, traditional serrated adenoma, or miscellaneous. Hyperplastic polyps, sessile serrated adenomas/polyps, and traditional serrated adenomas were grouped as serrated lesions, as their endoscopic appearance is similar (mostly sessile or flat morphology, and all considered as nonbleeding lesions) [20]. Dysplasia was defined as either low grade or high grade. Advanced adenoma was defined as any adenoma $\geq 10 \mathrm{~mm}$ or an adenoma with $>25 \%$ villous component or with high grade dysplasia. Cancers were staged according to the 7th edition of the American Joint Committee on Cancer classification [21,22].

Data on location, size, macroscopic aspect, morphology, method of polypectomy, and endoscopic assessment of completeness of polypectomy were recorded for all lesions detected during colonoscopy.

\section{Analyses}

All screening participants who underwent colonoscopy were classified into subgroups, based on characteristics of the most advanced lesion detected during colonoscopy. Lesions were classified in the following order: carcinomas, advanced adenomas, nonadvanced adenomas, serrated lesions, and no colonic lesions. Subgroups were defined based on histopathology, morphology, size, and location.

Histopathology subgroups were defined as: carcinoma, advanced adenomas, nonadvanced adenomas, serrated lesion, and no colonic lesions. Morphology subgroups were defined as flat, sessile, or pedunculated, as described by the Paris Classification for Colonic Lesions [23]. Size categories were: $1-5 \mathrm{~mm}, 6-9 \mathrm{~mm}$, $\geq 10 \mathrm{~mm}$. Location of colonic lesions was defined as proximal or distal to the splenic flexure. Location subgroups were alternatively defined by colonic segment (rectum, sigmoid, descending colon, splenic flexure, transverse colon, hepatic flexure, ascending colon, and cecum).

In each of these subgroups, participants were classified only once (only the most advanced lesion detected per participant was analyzed); synchronous or metachronous lesions were not evaluated. Carcinomas were excluded from the morphology subgroups, as these lesions often present with a different morphology from regular polyps (e.g. depressed or ulcerated lesions).

The mean and median FIT result was calculated in $\mu \mathrm{g} \mathrm{Hb} / \mathrm{g}$ feces for each of these subgroups. As FIT results are non-negative, a logarithmic transformation was first applied to better approach normality. Statistically significant differences in the mean results were tested between each set of subgroups (histopathology, morphology, size, and location) using one-way analysis of variance. Post hoc analyses were performed, applying a Bonferroni correction, to evaluate pairwise differences between subgroups. A multiple linear regression model was used to evaluate the conditional effects of polyp size, polyp morphology, and polyp location on the FIT result, adjusted for the other polyp features. The FIT result was the dependent variable, and the polyp characteristics were the independent variables.

In addition, proportions of the respective colonic lesions identified in the FIT-positive individuals from FIT-based screening and in the primary colonoscopy screening participants of the primary colonoscopy screening trial were calculated. Those proportions were compared using the chi-squared test.

\section{Results}

\section{$\nabla$}

Polyp characteristics in FIT-based screening

In three consecutive rounds of FIT-based screening, 877 FIT-positive individuals underwent colonoscopy. FIT results ranged from 10 to $812 \mu \mathrm{g} \mathrm{Hb} / \mathrm{g}$ feces; the mean amount of hemoglobin detected was $74 \mu \mathrm{g} \mathrm{Hb} / \mathrm{g}$ feces. $\odot$ Table 1 shows the mean and median FIT result in subgroups defined by polyp histopathology, morphology, size, and location (log-transformed FIT values were used for the analyses).

Mean FIT results differed significantly between the subgroups defined by histopathology findings $(P<0.001)$. Post hoc analyses showed that screenees with an advanced adenoma or carcinoma had a significantly higher mean FIT result than screenees with no colorectal lesions $(P=0.006$ and $P<0.001$, respectively), nonadvanced adenomas (both $P<0.001$ ) or serrated lesions only $(P=0.001$ and $P<0.001$, respectively). The mean FIT result of individuals with carcinomas was significantly higher than the FIT results of those with advanced adenomas $(P<0.001)$. Compared with individuals without polyps at colonoscopy, no differences in hemoglobin levels were found for those with serrated lesions $(P=1.0)$ or nonadvanced adenomas $(P=0.630)$. An additional analysis was performed among the 85 individuals with a serrated lesion. No significant differences in FIT results were found between serrated lesions of different subtypes or size $(P=0.13$; see Table e2, available online).

The morphology of most carcinomas was characterized as "different" or "stenotic." Excluding carcinomas, 592 lesions were included in the analysis of polyp morphology. Mean FIT results differed significantly between subgroups $(P<0.001)$ ( $\Theta$ Table 1 , - Fig. 3). Pairwise differences were significant between pedunculated polyps and polyps with sessile or flat morphology $(P<0.001$ and $P=0.001)$.

Size was known for 636 of the 651 lesions. Lesions of $10 \mathrm{~mm}$ or larger had significantly higher FIT results than small polyps and diminutive polyps $(P<0.001)$ : 260 lesions $(41 \%)$ were $\geq 10 \mathrm{~mm}$, and these had a significantly higher FIT result than smaller polyps $(6-9 \mathrm{~mm})$ and diminutive polyps $(1-5 \mathrm{~mm})(P<0.001)$. Hemoglobin concentrations were higher for polyps located in the distal colon than in the proximal colon. Analysis of polyp location specified for different colon segments also showed a significant difference ( $P=0.001)$ (see Table e3, available online). This could be explained by higher FIT results in participants with lesions located in the sigmoid.

\section{Multiple linear regression}

Multiple linear regression was performed to evaluate whether polyp size, polyp morphology, and polyp location, respectively, were associated with differences in mean FIT results, when taking the other characteristics into account. The analysis showed that polyp location was no longer significantly associated with the amount of fecal hemoglobin when adjusting for the polyp size and morphology $(P=0.32)$. Polyp size and polyp morphology, however, remained significantly associated with the FIT result when adjusted for other polyp characteristics $(P<0.001$ and $P=$ 0.005 , respectively).

\section{Polyp characteristics in colonoscopy-based screening}

In the primary colonoscopy trial, 1426 participants underwent a colonoscopy, of whom 1256 (88\%) also completed a FIT test [16]. FIT results ranged from 0 to $670 \mu \mathrm{Hb} / \mathrm{g}$ feces, with a mean of $9 \mu \mathrm{H} \mathrm{Hb} / \mathrm{g}$ feces. 
Table 1 Fecal immunochemical test results and characteristics of the most advanced lesion detected at colonoscopy.

\begin{tabular}{|c|c|c|c|c|c|c|c|c|}
\hline & \multicolumn{4}{|c|}{ FIT-based screening group } & \multicolumn{4}{|c|}{ Colonoscopy screening group } \\
\hline & \multirow[t]{2}{*}{ n (\%) } & \multicolumn{2}{|c|}{$\begin{array}{l}\text { Absolute FIT result, } \\
\mu \mathrm{g} \mathrm{Hb} / g \text { feces }\end{array}$} & \multirow[t]{2}{*}{$P^{1}$} & \multirow[t]{2}{*}{ n (\%) } & \multicolumn{2}{|c|}{$\begin{array}{l}\text { Absolute FIT result, } \\
\mu \mathrm{g} \mathrm{Hb} / \mathrm{g} \text { feces }\end{array}$} & \multirow[t]{2}{*}{$P^{1}$} \\
\hline & & Mean (SD) & Median (IQR) & & & Mean (SD) & Median (IQR) & \\
\hline Total & 877 & $74(119)$ & $26(15-69)$ & & 1256 & $9(47)$ & $0(0-1)$ & \\
\hline Histopathology & 877 & & & $<0.001$ & 1256 & & & $<0.001$ \\
\hline No colorectal lesions & $226(26)$ & $66(111)$ & $23(14-53)$ & & $685(55)$ & $3(20)$ & $0(0-0)$ & \\
\hline Serrated lesion ${ }^{2}$ & $85(10)$ & $46(77)$ & $20(15-40)$ & & $191(15)$ & $5(45)$ & $0(0-1)$ & \\
\hline Nonadvanced adenoma & $195(22)$ & $50(95)$ & $19(14-37)$ & & $261(21)$ & $5(20)$ & $0(0-2)$ & \\
\hline Advanced adenoma & $332(38)$ & $87(127)$ & $34(17-93)$ & & $111(9)$ & $49(118)$ & $2(0-27)$ & \\
\hline Carcinoma & $39(4)$ & $199(185)$ & $136(57-307)$ & & $8(<1)$ & $155(150)$ & $128(25-241)$ & \\
\hline Polyp morphology & 592 & & & $<0.001$ & 531 & & & $<0.001$ \\
\hline Flat & $66(11)$ & $48(66)$ & $21(14-53)$ & & $40(7)$ & $2(3)$ & $0(0-2)$ & \\
\hline Sessile & $318(54)$ & $54(99)$ & $20(14-47)$ & & $408(77)$ & $10(51)$ & $0(0-2)$ & \\
\hline Pedunculated & $208(35)$ & $100(139)$ & $41(19-109)$ & & $83(16)$ & $42(112)$ & $2(0-20)$ & \\
\hline Polyp size & 636 & & & $<0.001$ & 569 & & & \\
\hline$\geq 10 \mathrm{~mm}$ & $260(41)$ & $109(146)$ & $47(20-125)$ & & $100(18)$ & $52(121)$ & $2(0-34)$ & $<0.001$ \\
\hline $6-9 \mathrm{~mm}$ & $110(17)$ & $58(104)$ & $21(14-43)$ & & $75(13)$ & $14(50)$ & $0(0-5)$ & \\
\hline $1-5 \mathrm{~mm}$ & $266(42)$ & $54(96)$ & $21(15-45)$ & & $394(69)$ & $7(43)$ & $0(0-1)$ & \\
\hline Polyp location & 647 & & & $<0.001$ & 571 & & & 0.001 \\
\hline Proximal (to splenic flexure) & $227(35)$ & $56(91)$ & $22(15-53)$ & & $255(45)$ & $11(54)$ & $0(0-2)$ & \\
\hline Distal (including splenic flexure) & $420(65)$ & $88(135)$ & $31(16-92)$ & & $316(55)$ & $20(75)$ & $0(0-4)$ & \\
\hline
\end{tabular}

FIT, fecal immunochemical test; IQR, interquartile range.

${ }^{1}$ One-way analysis of variance.

${ }^{2}$ Hyperplastic polyp, sessile serrated adenoma/polyp or traditional serrated adenoma

Mean FIT results differed significantly depending on the histopathology results $(P<0.001)(\Theta$ Table 1$)$. A post hoc analysis showed differences between subgroups that were comparable to those in the FIT-positive individuals in FIT-based screening, with significant differences between screenees with advanced adenomas and carcinomas compared with those without colorectal lesions (both $P<0.001$ ). Participants with nonadvanced adenomas had significantly higher FIT results than those without colorectal lesions $(P=0.001)$. Of the 571 lesions detected in the colonoscopy screenees, morphology could be assessed in 538, and 531 remained after exclusion of the 7 carcinomas with known morphology. Mean FIT results differed significantly between groups $(P<0.001)$.

Size was known for 569 of the 571 lesions. FIT results were significantly different among differently sized polyps. Of the 571 colorectal lesions, 255 were located proximal to the splenic flexure. Distally located lesions had a significantly higher hemoglobin concentration than proximally located lesions $(P=0.001)$, reflecting results in the FIT-based screening.

\section{FIT-positive screening vs. colonoscopy screening}

The distribution of colonic lesions was compared between the FIT-positive individuals in the FIT-based screening rounds and the participants undergoing primary colonoscopy screening. As expected, there were more advanced adenomas in the FIT-positive individuals than in the primary colonoscopy screenees (38\% vs. $8 \%$ ), as well as more carcinomas ( $4 \%$ vs. $<1 \% ; P<0.001$ ) ( $\odot$ Table 4). There was no difference in the number of screening participants with nonadvanced adenomas ( $22 \%$ vs. $20 \%$, respectively; $P=0.32$ ).

\section{Discussion}

This study showed that the mean FIT result for FIT-positive screening individuals with advanced adenomas and carcinomas is significantly higher than the mean in those with nonadvanced adenomas, serrated lesions, and those with no colorectal lesions. Screening individuals with pedunculated polyps had a higher mean FIT result than those with sessile and flat lesions, and screenees with polyps $\geq 10 \mathrm{~mm}$ had a higher FIT result than those with smaller lesions.

As expected, significantly more participants had advanced adenomas and carcinomas in the FIT-positive group than in the screening colonoscopy group. The number of nonadvanced adenomas, however, was comparable in the two groups.

Some limitations should be acknowledged. The analyses were based on the most advanced lesion detected during colonoscopy, and no adjustment was made for possible additional relevant pathology. Previous trials have evaluated the association between the occurrence of multiple adenomas and the amount of fecal hemoglobin $[9,10]$; this could be relevant because multiple nonadvanced adenomas, as weak sources of bleeding, may together produce a positive FIT result. Such analyses were not performed in the current study, as it was believed that the absolute hemoglobin concentration is largely the result of the most advanced lesion, and the hypothesis was that more advanced lesions would show a higher FIT result. A previous cross-sectional study in symptomatic patients who were referred for colonoscopy reported that a higher T stage in CRC is associated with higher FIT values [8]. In the current study, only 47 carcinomas were detected in total, which prohibits precise analysis of the effect of stage on FIT values.

The first round of the FIT-based screening program started in 2006, and this allowed analysis of 877 FIT-positive individuals. 

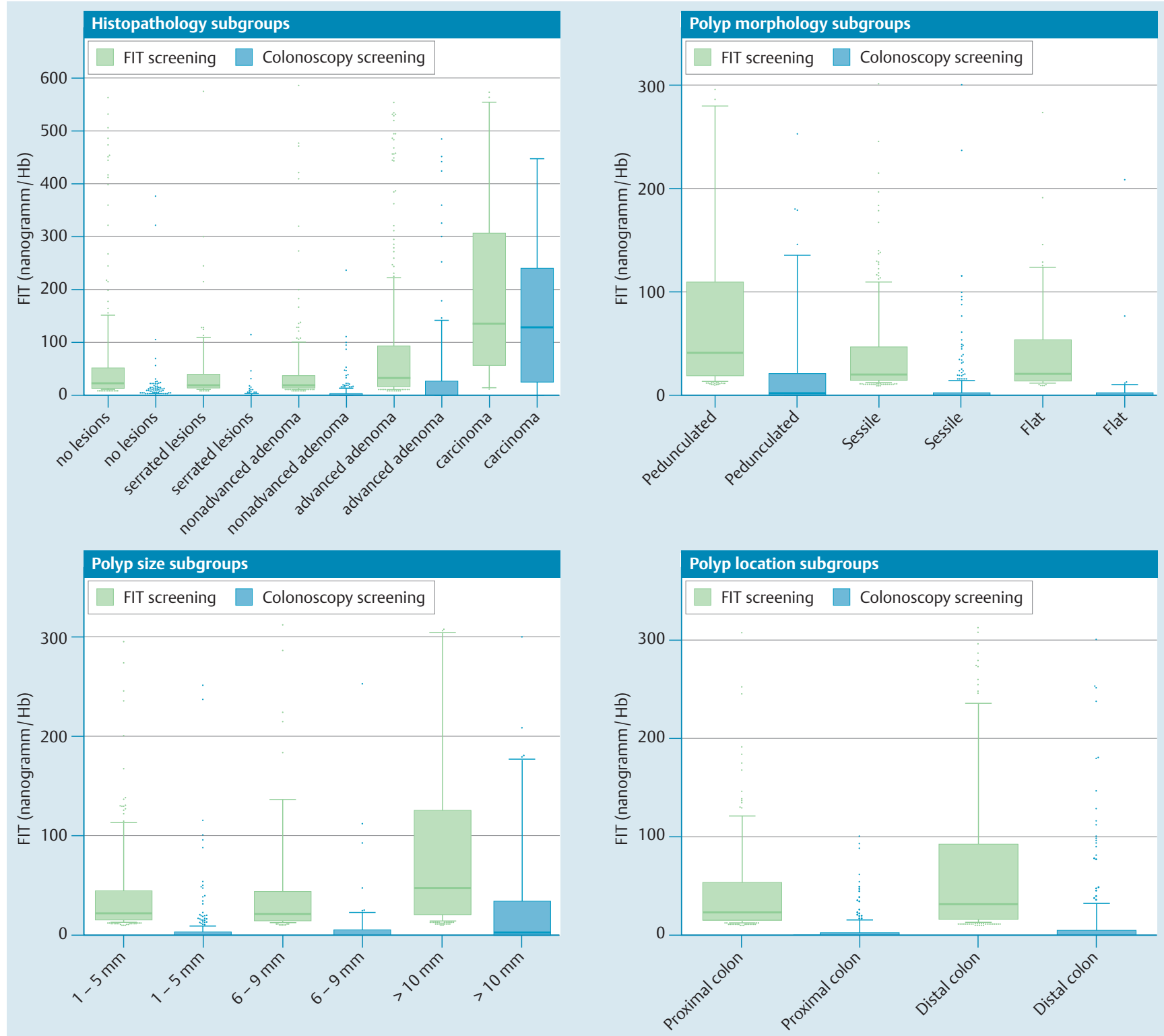

Fig. 3 Boxplots showing the differences between histopathology, morphology, size, and location subgroups and 25th percentiles fecal immunochemical test (FIT) in $\mathrm{ng} \mathrm{Hb} / \mathrm{mL}$ buffer.

Experienced research staff registered all detected and removed polyps during the colonoscopies in both screening programs. In both screening pilots, most colonoscopies were performed by the same endoscopists. All polyps were sent to pathology for assessment, which allowed the analyses in the current study. Although both screening populations underwent a different screening route, they were all asymptomatic, comparable in age, lived largely in the same region, and all had a similar socioeconomic status.

Previous trials have also reported higher fecal hemoglobin levels in patients with advanced adenomas and carcinomas compared with patients with nonadvanced adenomas or no neoplasia [9, $10,24]$. Two studies showed that individuals with nonadvanced adenomas $>6 \mathrm{~mm}$ had a higher FIT result than those with a normal examination $[9,10]$. The current findings did not confirm this, as the mean FIT result in the group of FIT-positive individuals with nonadvanced adenomas of any size was not higher than in individuals without lesions. Digby et al. demonstrated an association between fecal hemoglobin and the severity of neoplastic disease in a large FIT-positive cohort undergoing colonoscopy [12]. A limitation of this study was that the distributions of fecal hemoglobin could not be fully assessed, as the upper analytical limit was $1000 \mathrm{ng} \mathrm{Hb} / \mathrm{mL}$ buffer [12]. The current study demonstrated quantitative results of all FIT-positive individuals, with a cutoff level of $50 \mathrm{ng} \mathrm{Hb} / \mathrm{mL}$ instead of $400 \mathrm{ng} \mathrm{Hb} / \mathrm{mL}$ buffer. The difference in cutoff level and analytical limit may explain the difference in findings between the Digby study and the current study, as the latter describes a higher incidence of nonadvanced adenomas and hyperplastic polyps. Whereas Digby showed that lesion size was the only characteristic significantly related to fecal hemoglobin, the current study demonstrated that polyp morphology was also significantly related to the FIT result. Another limitation of the Digby study is that only FIT-positive participants underwent colonoscopy and could therefore be assessed [12]. To overcome this limitation, the current study also assessed FIT results from participants from an asymptomatic colonoscopy 


\begin{tabular}{|c|c|c|c|}
\hline & $\begin{array}{l}\text { FIT-based screening } \\
\text { group } \\
\mathrm{N}=877\end{array}$ & $\begin{array}{l}\text { Primary colonoscopy } \\
\text { screening group }{ }^{1} \\
N=1426\end{array}$ & $P$ \\
\hline \multicolumn{4}{|l|}{ Histopathology, n (\%) } \\
\hline No colorectal lesions & $226(26)$ & $778(55)$ & - \\
\hline Serrated lesions ${ }^{2}$ & $85(10)$ & $226(16)$ & $<0.001$ \\
\hline Nonadvanced adenomas & $195(22)$ & $292(20)$ & 0.32 \\
\hline Advanced adenomas & $332(38)$ & $121(8)$ & $<0.001$ \\
\hline Carcinomas & $39(4)$ & $9(1)$ & $<0.001$ \\
\hline
\end{tabular}

Table 4 Most advanced lesions detected at colonoscopy.

FIT, fecal immunochemical test.

1 Including both patients who performed colonoscopy and FIT and those who only performed colonoscopy.

${ }^{2}$ Hyperplastic polyp or sessile serrated adenoma/polyp

screening trial. Results demonstrated that the same associations between polyp characteristics and FIT results could be evaluated, when FIT results below the cutoff level of $50 \mathrm{ng} \mathrm{Hb} / \mathrm{mL}$ ("negative FIT results") were also taken into account.

FIT results in pedunculated polyps were higher than in sessile lesions. A previous study evaluating FIT levels and polyp features suggested that pedunculated lesions are often larger than sessile polyps, but the authors of that study did not detect a significant difference in FIT results between polyps of different sizes [9]. Haug et al. demonstrated a higher sensitivity of FIT for the detection of advanced neoplasia in the left vs. right colon, and they also found a significantly higher incidence of pedunculated shaped lesions in the left colon [25]. Although the authors only evaluated the association between FIT results and advanced neoplasia, they also found a higher median fecal hemoglobin level in pedunculated lesions. In their logistic regression analysis, pedunculated shape was statistically significantly associated with test sensitivity even when adjusted for size [25]. This is in line with the current results; in the multiple variable analyses, polyp size (higher FIT results for lesion of $\geq 10 \mathrm{~mm}$ ) and polyp morphology (higher FIT results for pedunculated lesions) remained significantly associated with the FIT result when adjusted for other polyp characteristics. Carcinomas were excluded from the morphology analyses, as these lesions often present with different morphology from the regular polyp morphology according to the Paris classification [23]. It would be interesting to evaluate whether fecal hemoglobin levels differ with the shape of carcinomas, and to examine whether, for example, depressed carcinomas are more easily missed by FIT. Unfortunately, the number of carcinomas in the current study $(n=39)$ was too small for this type of analysis; larger additional studies are needed to evaluate this further.

Whereas individuals in the FIT-based screening group who had the most advanced lesion in the proximal colon had a significantly lower FIT result compared with that in individuals with distally located adenomas, this was not observed in two other studies [9, 10]. However, polyp location was no longer significantly associated with fecal hemoglobin level when adjustment was made for the size and morphology, which is in line with previous studies $[9,10]$. Haug et al. reported a higher sensitivity of FIT for distally located lesions [25]. The authors hypothesized that this might be caused by less hemoglobin degradation from left-sided lesions, and to difference in stool consistency in the left- vs. the rightsided colon and more homogeneously distributed blood in the stools from the right side. As described above, the authors detected more pedunculated lesions in the distal colon. Although in the current study the significant association between polyp location and FIT result disappeared after adjustment for polyp size and morphology, the morphology of polyps might be of larger influence of the fecal hemoglobin level than location.
To our knowledge, this is the first study to analyze differences in FIT results for the different subtypes of serrated lesions. In two other trials, serrated adenomas were regarded as adenomatous, whereas all serrated lesions in the current study were regarded as separate findings $[9,10]$. As the mean FIT result in individuals with serrated lesions was not higher than in those without lesions, one might conclude that serrated lesions are coincidental, nonbleeding findings in FIT-positive individuals, and that FIT does not seem to detect or differentiate between serrated lesions. Regrettably no analyses could be performed on dysplasia in serrated lesions, because the first screening round was performed in 2006 and, at that time, dysplasia could not be assessed as reliably as it is today. As the mean FIT result in screenees with an advanced adenoma was approximately $87 \mu \mathrm{g} \mathrm{Hb} / \mathrm{g}$ feces in the FITbased screening group and $49 \mu \mathrm{g} \mathrm{Hb} / \mathrm{g}$ feces in the colonoscopy group, one could argue that the FIT positivity cutoff level of $10 \mu \mathrm{g} \mathrm{Hb} / \mathrm{g}$ feces should be increased. In countries where colonoscopy capacity is limited, increasing the cutoff level within the screening trial will decrease the number of unnecessary colonoscopies in participants with a false-positive FIT and only nonadvanced lesions. However, as previous studies have shown, this would inevitably lead to missed carcinomas and advanced adenomas $[5,16]$. As an alternative, the absolute FIT result could be incorporated into a risk model for advanced neoplasia. A recent publication demonstrated that individual FIT concentrations are predictive for the presence of colorectal neoplasia [24]. Our research group previously described several risk factors that were related to a false-negative or false-positive FIT result $[26,27]$. Using a combination of the absolute FIT result and other risk factors to determine whether individuals are invited for colonoscopy following FIT-based screening, rather than using FIT positivity alone, could increase sensitivity without a loss in specificity, potentially improving the effectiveness of CRC programs [16,27]. Although screening participants with advanced adenomas and carcinomas had elevated FIT results, FIT-positive individuals with nonadvanced adenomas did not have a higher FIT result than individuals without colorectal lesions. In addition, the number of screenees with nonadvanced adenomas was comparable in FIT-positive individuals and among primary colonoscopy screening participants. This combination of findings suggests that nonadvanced adenomas are coincidental findings in FIT-positive individuals, reflecting simply the background prevalence of these lesions in the age group targeted for screening. The fact that FIT does not seem to detect these lesions accurately can be regarded as an advantage. FIT-based screening aims to select participants with an increased risk of advanced lesions; only these individuals are invited for colonoscopy. As screening with FIT is typically repeated at fixed intervals, and given the slow progression rate of adenomas, participants with nonadvanced adenomas are likely 
to be invited for colonoscopy in the future, but only when their adenomas have advanced to larger, bleeding lesions.

\section{Competing interests: None}

\section{Institutions}

${ }^{1}$ Department of Gastroenterology and Hepatology, Academic Medical Center, Amsterdam, The Netherlands

2 Department of Clinical Epidemiology, Biostatistics and Bioinformatics, Academic Medical Center, Amsterdam, The Netherlands

${ }^{3}$ Department of Clinical Chemistry, Academic Medical Center, Amsterdam, The Netherlands

${ }^{4}$ Department of Gastroenterology and Hepatology, Flevoziekenhuis Almere, The Netherlands

${ }^{5}$ Department of Gastroenterology and Hepatology, Erasmus Medical Center, Rotterdam, The Netherlands

\section{Acknowledgment}

\section{$\nabla$}

The study was supported by a grant from The Netherlands Organization for Health Research and Development of the Dutch Ministry of Health (ZonMW 12010095420). FIT tests and reagents needed for laboratory assessment for the 3 rd pilot round of FIT screening were provided by Eiken Chemical Co., LTD., Japan. The sponsor was not involved in the study.

\section{References}

1 Zavoral M. Colorectal cancer screening in Europe. World J Gastroenterol 2009; 15: 5907

2 Libby G, Brewster DH, McClements PL et al. The impact of populationbased faecal occult blood test screening on colorectal cancer mortality: a matched cohort study. Br J Cancer 2012; 107: 255-259

3 Van Rossum LG, Van Rijn AF, Laheij RJ et al. Random comparison of guaiac and immunochemical fecal occult blood tests for colorectal cancer in a screening population. Gastroenterology 2008; 135: 820

4 Hol L, Wilschut JA, van Ballegooijen M et al. Screening for colorectal cancer: random comparison of guaiac and immunochemical faecal occult blood testing at different cut-off levels. Br J Cancer 2009; 100: 1103 1110

5 van Rossum LG, van Rijn AF, Laheij RJ et al. Cutoff value determines the performance of a semi-quantitative immunochemical faecal occult blood test in a colorectal cancer screening programme. Br J Cancer 2009; 101: 1274-1281

6 Van Roon AHC, Hol L, Van Vuuren AJ et al. Are fecal immunochemical test characteristics influenced by sample return time? A populationbased colorectal cancer screening trial. Am J Gastroenterol 2012; 107: 99-107

7 Fraser CG, Allison JE, Halloran SP et al. A proposal to standardize reporting units for fecal immunochemical tests for hemoglobin. J Natl Cancer Inst 2012; 104: 810-814

8 van Turenhout ST, van Rossum LG, Oort FA et al. Similar fecal immunochemical test results in screening and referral colorectal cancer. World J Gastroenterol 2012; 18: 5397-5403

9 Rozen P, Levi Z, Hazazi R et al. Identification of colorectal adenomas by a quantitative immunochemical faecal occult blood screening test depends on adenoma characteristics, development threshold used and number of tests performed. Aliment Pharmacol Ther 2009; 29: 906 917
10 Levi Z, Rozen P, Hazazi R et al. A quantitative immunochemical fecal occult blood test for colorectal neoplasia. Ann Intern Med 2007; 146: $244-255$

11 Cubiella J, Castro I, Hernandez V et al. Characteristics of adenomas detected by fecal immunochemical test in colorectal cancer screening. Cancer Epidemiol Biomarkers Prev 2014; 23: 1884-1892

12 Digby J, Fraser CG, Carey FA et al. Faecal haemoglobin concentration is related to severity of colorectal neoplasia. J Clin Pathol 2013; 66: 415 419

13 Denters MJ, Deutekom M, Bossuyt PM et al. Lower risk of advanced neoplasia among patients with a previous negative result from a fecal test for colorectal cancer. Gastroenterology 2012; 142: 497 - 504

14 Stegeman I, de Wijkerslooth TR, Mallant-Hent RC et al. Implementation of population screening for colorectal cancer by repeated fecal immunochemical test (FIT): third round. BMC Gastroenterol 2012; 12: 73

15 Denters MJ, Deutekom M, Fockens P et al. Implementation of population screening for colorectal cancer by repeated fecal occult blood test in The Netherlands. BMC Gastroenterol 2009; 9: 28

16 De Wijkerslooth TR, Stoop EM, Bossuyt PM et al. Immunochemical fecal occult blood testing is equally sensitive for proximal and distal advanced neoplasia. Am J Gastroenterol 2012; 107: 1570-1578

17 Stoop EM, de Haan MC, de Wijkerslooth TR et al. Participation and yield of colonoscopy versus non-cathartic CT colonography in populationbased screening for colorectal cancer: a randomised controlled trial. Lancet Oncol 2012; 13: 55-64

18 De Wijkerslooth TR, de Haan MC, Stoop EM et al. Burden of colonoscopy compared to non-cathartic CT-colonography in a colorectal cancer screening programme: randomised controlled trial. Gut 2012; 61: $1552-1559$

19 Schlemper RJ, Riddell RH, Kato $Y$ et al. The Vienna classification of gastrointestinal epithelial neoplasia. Gut 2000; 47: 251-255

20 Snover DC, Ahnen DJ, Burt RW, Odze RD. Serrated polyps of the colon and rectum and serrated polyposis. In: Bosman FT, Carneiro F, Hruban $\mathrm{RH}$ et al. (eds.) WHO classification of tumours of the digestive system. France: Lyon: IARC; 2010: 160-165

$21 \mathrm{Kim}$ KH, Yang SS, Yoon YS et al. Validation of the seventh edition of the American Joint Committee on Cancer tumor-node-metastasis (AJCC TNM) staging in patients with stage II and stage III colorectal carcinoma: analysis of 2511 cases from a medical centre in Korea. Colorectal Dis 2011; 13: e220-226

22 Edge SB, Compton CC. The American Joint Committee on Cancer: the 7th edition of the AJCC cancer staging manual and the future of TNM. Ann Surg Oncol 2010; 17: 1471 - 1474

23 Endoscopic Classification Review Group. Update on the Paris classification of superficial neoplastic lesions in the digestive tract. Endoscopy 2005; 37: $570-578$

24 Liao C-S, Lin $Y$-M, Chang $H-C$ et al. Application of quantitative estimates of fecal hemoglobin concentration for risk prediction of colorectal neoplasia. World J Gastroenterol 2013; 19: 8366-8372

25 Haug $U$, Kuntz KM, Knudsen $A B$ et al. Sensitivity of immunochemical faecal occult blood testing for detecting left- vs right-sided colorectal neoplasia. Br J Cancer 2011; 104: 1779-1785

26 Stegeman I, de Wijkerslooth TR, Stoop EM et al. Risk factors for false positive and for false negative test results in screening with fecal occult blood testing. Int J Cancer 2013; 133: 2408-2414

27 Stegeman I, de Wijkerslooth TR, Stoop EM et al. Combining risk factors with faecal immunochemical test outcome for selecting CRC screenees for colonoscopy. Gut 2014; 63: 466-471

Fig. e1, e2 and Table e2, e3

online content viewable at:

http://dx.doi.org/10.1055/s-0034-1392412 